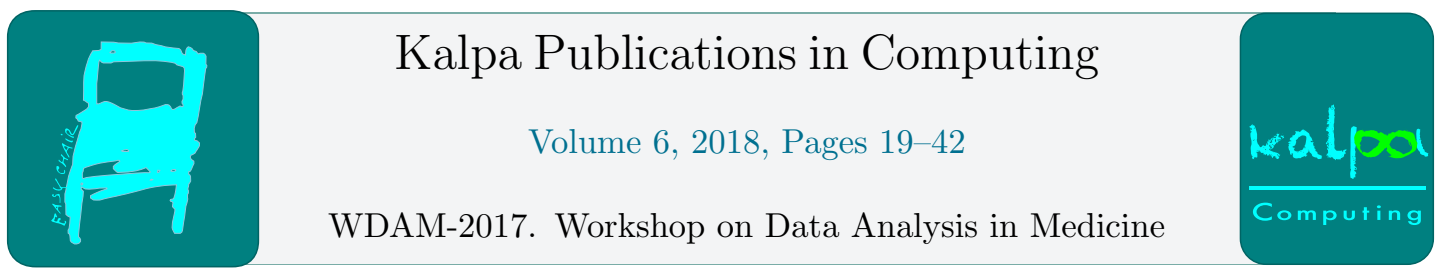

\title{
Consolidated mathematical growth Model of Breast Cancer CoMBreC
}

\author{
Ella Ya. Tyuryumina ${ }^{1}$ \\ International Laboratory for Intelligent Systems and Structural Analysis, \\ at National Research University Higher School of Economics, Moscow, Russia \\ etyuryumina@hse.ru
}

\begin{abstract}
This paper is devoted to mathematical modelling of the progression considering stages of breast cancer. Given the relation between primary tumor (PT) and metastases (MTS), the problem of discovering breast cancer (BC) process seems to be twofold: firstly, it is important to describe the whole natural history of $\mathrm{BC}$ to understand the process as a whole; secondly, it is necessary to predict the period of a clinical MTS manifestation. In order to understand growth processes of $\mathrm{BC}$ on each stage CoMBreC was proposed as a new research tool. The CoMBreC is threefold: CoMPaS (stages I-II), CoM-III (stage III) and CoM-IV (stage IV). A new model rests on exponential growth model and complementing formulas. For the first time, it allows us to calculate different growth periods of PT and MTS in patients with/without lymph nodes MTS: 1) non-visible period for PT; 2) nonvisible period for MTS; 3 ) visible period for MTS. Calculations via CoMBreC correspond to survival data considering stage of BC. It may help to improve predicting accuracy of $\mathrm{BC}$ process using an original mathematical model referred to CoMBreC and corresponding software. Consequently, thesis concentrated on: 1) modelling the whole natural history of PT and MTS in patients with/without lymph nodes MTS; 2) developing adequate and precise CoMBreC that reflects relations between PT and MTS; 3) analysing the CoMBreC scope of application. The CoMBreC was implemented to iOS application as a new predictive tool: 1) is a solid foundation to develop future studies of BC models; 2) does not require any expensive diagnostic tests; 3 ) is the first predictor of survival in breast cancer that makes forecast using only current patient data.
\end{abstract}

Keywords: breast cancer, exponential growth model, mathematical model, metastases in lymph nodes, mortality, primary tumor, primary metastases, secondary metastases, survival.

\section{Introduction}

Breast Cancer (BC) is the most common cancer and also the leading cause of cancer mortality in women worldwide. BC accounts for about 20-25\% of all cancer types in women [22].

Finding algorithms to predict the growth of tumors has piqued the interest of researchers ever since the early days of cancer research. Many studies were carried out as an attempt to obtain reliable data on the natural history of $\mathrm{BC}$ growth. 
Mathematical modelling can play a very important role in the prognosis of BC. Various mathematical models were built to describe primary tumor (PT) growth and distant metastases (MTS) growth separately [20].

These days, the exponential, Gompertz, logistic and von Bertalanffy models are included into a group of classical mathematical models of PT growth [1]. For the breast data, the observed linear dynamics were best captured via exponential model that is situated for description of PT growth and, also, for secondary distant MTS growth [8,9,11-13,15-17, 19,30,34]. As for Gompertz and logistic models, they are used rarely in order to describe PT growth or secondary distant MTS growth $[5,18,32]$.

The duration of the period from the first $\mathrm{BC}$ cell to death refers to the natural history of BC [2]. Secondary distant MTS appear in various time in different organs. The interval between resection of PT and the first clinical manifestation of MTS (MTS free survival time or non-visible period) determined by PT size, the number of affected lymph nodes and MTS growth rate $[3,5,8,9,12,13,15-17,19,25,30,32-34]$. Survival (lifetime) is the period between the date of diagnosis (TNM staging system of BC) and the date of a patient death [22]. Survival among $\mathrm{BC}$ patients (\%) indicates the percentage of people in a study or treatment group who are alive for a given period of time after diagnosis. The percentage of patients who live at least 5-, 10-, 15-, 20-, 25- and 30-years after PT being treated is defined as 5-, 10-, 15-, 20-, 25- and 30-years observed survival rate of BC patients [22].

It is important to highlight that the natural history of $\mathrm{BC}$ continues after resection of PT. The next stage began with secondary distant MTS manifestation. When MTS reach the threshold volume, patients die from process of BC [1,4, 8, 9, 12,13,15-17, 19,21, 25,30,34]. All $\mathrm{BC}$ patients get a comprehensive treatment of $\mathrm{PT}$, therefore the whole natural history of $B C$ should include the period of growth secondary distant MTS:

1) the non-visible period of PT growth;

2) the visible period of PT growth, diagnostics and resection of PT;

3) the non-visible period of secondary distant MTS growth;

4) the visible period of secondary distant MTS growth, diagnostics, treatment and patient's death.

Given the relation between PT and MTS, the problem of discovering BC process seems to be twofold: firstly, it is important to describe the whole natural history of $B C$ to understand the process as a whole; secondly, it is necessary to predict the period of a clinical MTS manifestation. Yet, the papers available for this do not offer mathematical growth models of MTS that relate to TNM classification. That leads to the demand building a mathematical model that is based on exponential classical mathematical model, describes whole natural history of $B C$ and corresponds to TNM classification. Moreover, the latter aspect of the problem is reflected only by statistical tools that are available as open source. In other words, a patient provides diagnostic data to predictor, and the tool calculates MTS free period and survival according to statistical data. Consequently, it is necessary to create a predictor that makes prognosis of BC for a patient independently from statistical data, and requires no expensive diagnostic data. Thus, this research possesses a novelty since it is the first time the following tools for BC have been proposed: a) whole natural history of $B C$; b) mathematical growth model corresponding to TNM; c) non-statistical software tool for prediction of BC developing.

The goal of the research is to improve the prediction accuracy of $\mathrm{BC}$ process using the original Consolidated mathematical growth Model of Breast Cancer (CoMBreC) and implement with model in a software tool. 
Practical Value. As it turns out, a new software tool for prediction of BC developing can calculate more accurately: a) MTS free period; b) survival for stages I, II, III, IV of BC including primary MTS and MTS in lymph nodes. Moreover, the predictor can estimate a quality of treatment that was prescribed to a patient. Summarising: CoMBreC a) describes correctly PT growth of stages I, II, III, IV (T1-4N0-3M0-1); b) facilitates the understanding of the appearance period and inception of MTS.

\section{Theoretical background}

\subsection{Pathologic classification of Breast Cancer}

To avoid terminological ambiguities, we start with a short introduction into recalling some standard terms such as TNM staging system of BC (Tumor-Node-MTS) and Clinical Classification of Cancer [22] (Tab. 1,2).

Table 1: TNM staging system

\begin{tabular}{cccc}
\hline Stage & Parameter T & Parameter N & Parameter M \\
\hline I & T1 & N0 & M0 \\
II & T1, T2 & N0, N1 & M0 \\
III & any T & N1, N2, N3 & M0 \\
IV & any T & any N & M1 \\
\hline
\end{tabular}

Legend of Table 1:

$$
\begin{array}{lll}
\text { parameter } \mathrm{T}- & \text { size of PT: } \mathrm{T} 1=0.1 \mathrm{~cm}<\mathrm{d} \leq 2 \mathrm{~cm} ; \mathrm{T} 2=2 \mathrm{~cm}<\mathrm{d} \leq 5 \mathrm{~cm} ; \mathrm{T} 3 \\
& =\mathrm{d}>5 \mathrm{~cm} ; \mathrm{T} 4=\text { spread; } \\
\text { parameter } \mathrm{N}-\quad & \text { the number of affected lymph nodes: N0: } \mathrm{n}=0 ; \mathrm{N} 1: \mathrm{n}=1-3 ; \mathrm{N} 2: \\
& \mathrm{n}=4-9 ; \mathrm{N} 3: \mathrm{n}=10-18 ; \\
\text { parameter } \mathrm{M}-\quad \begin{array}{l}
\text { existence of distant MTS (lungs, bones, liver, etc): M0 }=\text { MTS not } \\
\text { exist; } \mathrm{M} 1=\text { MTS exist. }
\end{array}
\end{array}
$$

\subsection{Tumor doubling time}

Moiseenko (2002) [34] detected three following rate options of BC growth:

1) high rate - TDT $<90$ days $(20 \%)$; 2$)$ middle rate -90 days $\leq$ TDT $\leq 110$ days $(60 \%)$;

3 ) low rate - TDT $>110$ days $(20 \%)$.

\section{Literature review}

\subsection{History of mathematical models}

Various forms of genetic instability and increased mutation rate can lead to a development of malignancies. At this stage, we can dwell upon a review of classical mathematical growth models of PT and secondary distant MTS of BC, such as the exponential model [4], the von Bertalanffy model [29], the Gompertz model and logistic model [20].

The attempts of PT growth formalisation were pioneered by three different scientists in 1930 independently; namely Casey, Shreck and Mayneord built surface growth and "sigmoid" 
Table 2: Clinical Classification of Cancer

\begin{tabular}{|l|c|c|c|}
\hline Stage 0 & T0 & N0 & M0 \\
\hline Stage I & T1 & N0 & M0 \\
\hline Stage IIA & T0 & N1 & M0 \\
& T1 & N1 & M0 \\
& T2 & N0 & M0 \\
\hline Stage IIB & T2 & N1 & M0 \\
& T3 & N0 & M0 \\
\hline Stage IIIA & T0 & N2 & M0 \\
& T1 & N2 & M0 \\
& T2 & N2 & M0 \\
& T3 & N1, N2 & M0 \\
\hline Stage IIIB & T4 & N0, N1, N2 & M0 \\
\hline Stage IIIC & Any T & N3 & M0 \\
\hline Stage IV & Any T & Any N & M1 \\
\hline
\end{tabular}

growth models. I.A. Rodriguez-Brenes et al. [20] provides a complete overview of the history of mathematical models of PT growth.

\subsection{Comparative analysis of classical mathematical models of PT growth}

V.P. Collins et al. (1956) [4] have proposed that linear exponential growth, determined by measurement of pulmonary MTS and expressed as 'Tumor Volume Doubling Time' (TVDT), is a characteristic of individual cancer, governing the duration before and after diagnosis.

L.Von Bertalanffy (1957) [29] has built a mathematical model, as an attempt to connect metabolism to growth.

A.K. Laird (1964) [14] has introduced an idea that an exact mathematical description of the model of tumor cell proliferation is given by a Gompertz.

J.A. Spratt et al. (1993) $[23,24]$ have concerned that BC growth rates can be calculated using a form of the logistic equation for a large group of patients undergoing routine screening mammography.

The rate of tumor growth is directly proportional to the tumor size and depends on a number of nutrients.

Concerning that the tumor reaches a maximum size at the certain time, mathematical models, such as Gompertz, von Bertalanffy and logistic, describe a slowing growth rate. Consequently, these models are common S shape (sigmoid curve). A sigmoid curve is a bounded differentiable real function that is defined for all real input values and has a positive derivative at each point $[14,20,23,24,29]$.

Nowadays, a group of classical mathematical models of PT growth consists of the exponential (with or without free initial volume), Gompertz, logistic and von Bertalanffy models [1]. For data on $\mathrm{BC}$, the observed linear dynamics is best captured by exponential model that is situated for description of PT growth and, also, for secondary distant MTS growth $[8,9,12,13,15-17$, $19,25,30,34]$. As for Gompertz and logistic, models are used rarely in a description of PT and secondary distant MTS growth processes [5, 18, 32]. 
I.A. Rodriguez-Brenes et al. (2013) [20] have provided a complete overview of the history of mathematical models of PT growth.

S. Benzekry et al. (2014) [1] have completed the experimental studying about the growth of primary BC and lung cancer. As researchers indicate, both of the Gompertz and exponential growth model describes primary $\mathrm{BC}$ most voraciously.

\subsection{Mathematical models of secondary distant MTS}

After diagnostic of PT, the multi-modality treatment takes place: surgery (lumpectomy, unilateral or bilateral mastectomy), radiotherapy and chemotherapy. Yet, secondary distant MTS appear in different organs, considering the size of PT over time [5]. The metastatic process of tumor cells depends on the number of circulating tumor cells (CTCs) in blood. We may define four levels of CTCs [5]: 1) 0 - almost healthy people; 2) 1-4 tumor cells in $7.5 \mathrm{ml}$ of blood - the lowest level of metastatic process; 3) 5-24 tumor cells - the middle level of metastatic process; 4) more than 25 tumor cells - the most significant level of metastatic process.

The number of CTCs in blood depends on the size of PT, metastatic activity, and tumor cell aggressiveness. The dates of clinical manifestation after resection of PT (i.e. the duration of MTS-free period) is defined by the duration of the pre-clinical non-visible period of BC growth (doubling time) and the metastatic tumor rate $[5,6]$.

Researchers have been actively investigating the problem of formalisation of primary and secondary MTS. Yet, the papers available for this do not offer mathematical models that describe MTS growth relating with TNM classification. Consequently, a new mathematical model ought to be sough to agree with TNM classification, have higher prediction score and be independent from medical statistical parameters.

\subsection{Market of $\mathrm{BC}$ prediction software}

These days prediction of cancer care widely spread from screening to hospice. However, certain predictions problems take place: estimating a probability of appearance $\mathrm{PT}$, a distance between resection PT and appearance secondary MTS, and lifetime for last stages patients. To begin the study, it should be emphasised that many of the early stage cancers induce no symptoms. Therefore, a-propose treatment is recommended in order to increase patient survival. Under such circumstances, recent years have been tend to discover a new way of modelling risk prediction in cancer care. Some studies have also been performed developing statistical prediction algorithms that help evaluate the likelihood of an event personally [31]. At this point, it is important to mention that prediction models:

a) can demonstrate a greater accuracy than stage forecasting or risk groupings;

b) can process novel data such as genomic sets;

c) can suggest treatment methods.

\section{Methods}

\subsection{Consolidated mathematical growth model of PT and secondary MTS (CoMPaS)}

The natural history of BC continues after the resection of PT. Stage I-II (T1-2N0-1M0) means that secondary distant MTS appear after resection of PT [22]. Therefore if MTS reach the 
threshold volume, so patients die from progression of BC $[1,4,8,9,12,13,15-17,19,21,25,30,34]$. All of BC patients get the comprehensive treatment of PT, so the whole natural history of BC should include the period of growth secondary distant MTS (Fig. 1):

1) the non-visible period of PT growth (from the appearance of the first tumor cell (diameter $=10 \mu \mathrm{m})$ up to reaching of the visible size (diameter $=5 \mathrm{~mm})$ of $\mathrm{PT})$;

2) the visible period of PT growth, diagnostics and the resection of PT (from visible size (diameter $=1-5 \mathrm{~mm}$ ) of PT up to pre-surgery size);

3) the non-visible period of secondary distant MTS growth (from diagnosis (date of PT resection) up to visible size (diameter $=5 \mathrm{~mm}$ ) of at least one of MTS);

4) the visible period of secondary distant MTS growth (from diagnostic of visible size (diameter $=5 \mathrm{~mm})$ up to reaching lethal size $($ death $))$.

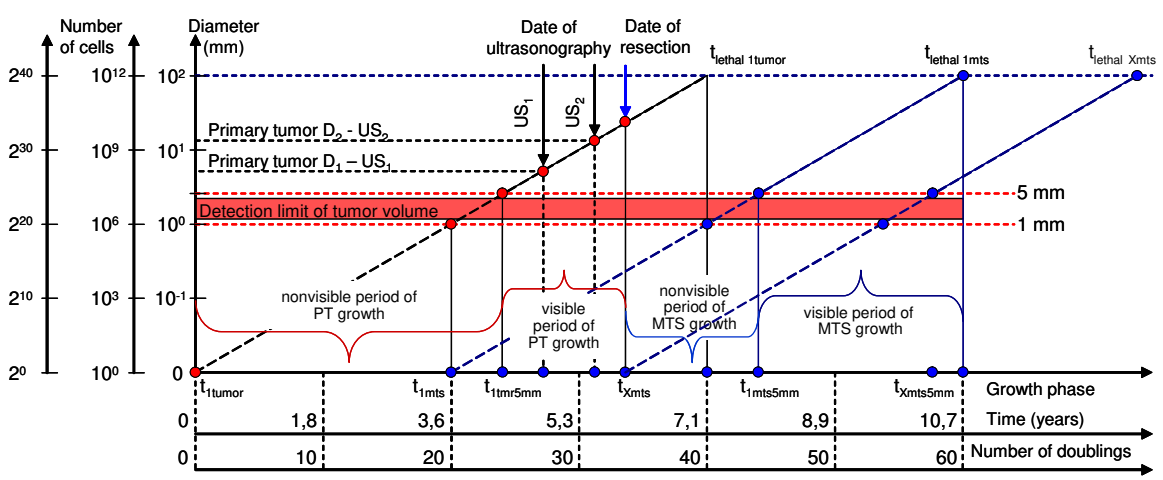

Figure 1: Scheme of the whole natural history of BC $[4,8,12,16,21,25-28]$. As it should be highlighted, the main feature is that model describes PT growth and secondary distant MTS growth as a whole (as indivisible dependent process). Ordinate (Y): Diameter of tumor (mm). Abscissa (X): Time (years). [27, 28]

Legend of Fig. 1 :

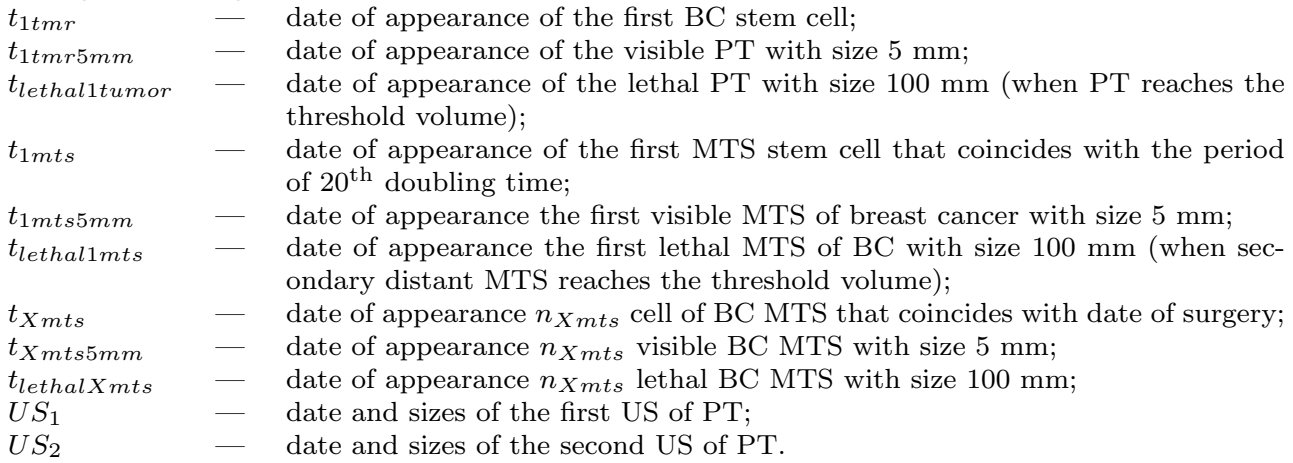

Under such circumstances, description of the whole natural history of $\mathrm{BC}$ requires upbuilding the consolidated mathematical growth model of PT and secondary distant MTS of BC.

It is important to define several admissions $(1-3[1,8,9,11-13,15,16,19,21,25,29,30,34])$ :

1) the exponential growth model was used to describe natural growth rate of primary BC; 
2) natural rate of secondary distant MTS is the same as natural growth rate of primary BC;

3) the period of appearance the first metastatic cell of secondary distant MTS coincides with the $20^{\text {th }}$ doubling of primary BC. It allows us to define the non-visible growth period of MTS and the initial period of non-visible MTS manifestation;

4) whole nature growth history of PT and secondary distant MTS is 60 doublings.

The CoMPaS may describe both of PT growth and secondary distant MTS growth considering histopathological classification. Also, the CoMPaS might facilitate the survival (lifetime) and, as a consequence, make predictions of a future metastatic manifestation after the resection of PT [26].

The CoMPaS was designed to predict how cancer is developed [26]. The CoMPaS rests on exponential growth model and consists of nonlinear and linear determined equations $[1,3,5,8$, 9,11-13,15-17, 19, 21, 25, 29, 30,33,34] (Fig. 2):

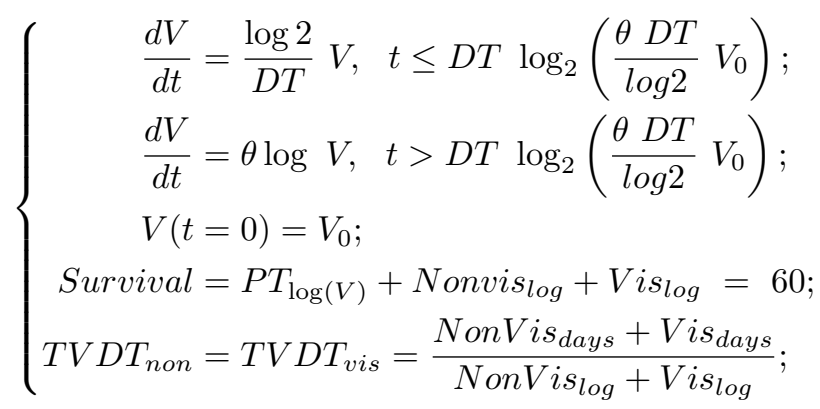

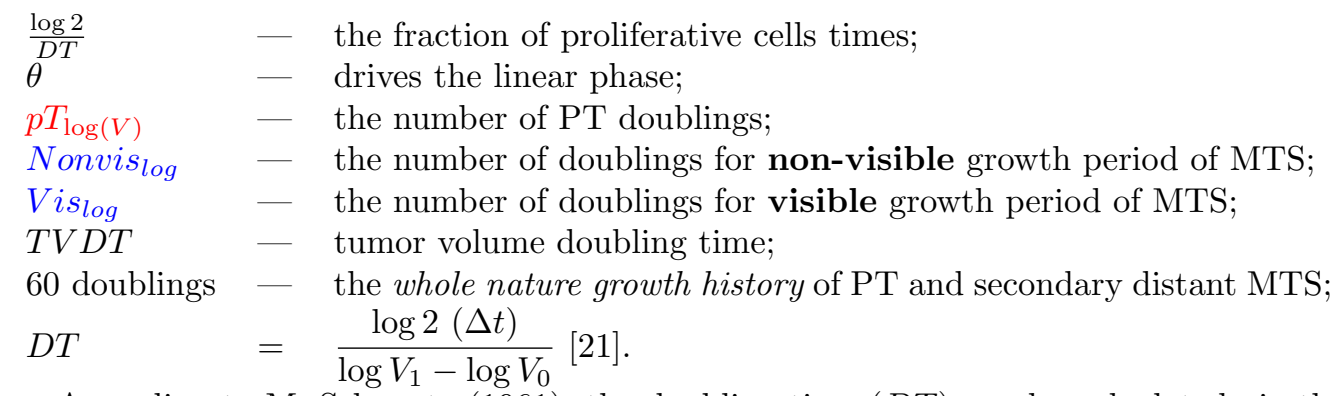

According to M. Schwartz (1961), the doubling time $(D T)$ can be calculated via the measurement of tumor volume $\left(V_{1}\right)$ at surgery $t_{1}$, the first measurement of tumor volume $\left(V_{0}\right)$ at diagnostic $t_{0}$ and the period between the measurements (days) $\left(\Delta t=t_{1}-t_{0}\right)$ [21].

$\mathrm{S}$. Friberg concerned, the tumor growth rate of $\mathrm{BC}$ is constant in visible period of tumor growth, in other words, diameter increases from $1 \mathrm{~mm}$ to $100 \mathrm{~mm}[1,3,5,8,9,11-13,15-17,19$, $21,25,29,30,33,34]$.

The diameter of the first tumor cell equals $10 \mu \mathrm{m}$. After 10th doubling the diameter of tumor equals $100 \mu \mathrm{m}$ (the number of cancer cells equals $10^{3}$ ), after $20^{\text {th }}$ doubling the diameter of tumor equals $1 \mathrm{~mm}$ (the number of cancer cells equals $10^{6}$ ), after $30^{\text {th }}$ doubling the diameter of tumor equals $10 \mathrm{~mm}$ (the number of cancer cells equals $10^{9}$ ), after $40^{\text {th }}$ doubling the diameter of tumor equals $103 \mathrm{~mm}$ (the number of cancer cells equals $10^{12}$ ).

The diameter of $\mathrm{PT}$ of $\mathrm{BC}$ that is more than $10 \mathrm{~cm}$ (the number of stem cells equals $10^{12}$ $10^{13}$ ), is threshold size of the tumor (lethal size). Such size promote BC patient death $[1,3,5$, $8,9,11-13,15-17,19,21,25,29,30,33,34]$. We may define following three tumor growth reasons:

1) the time of cycle dividing proliferating tumor cells; 


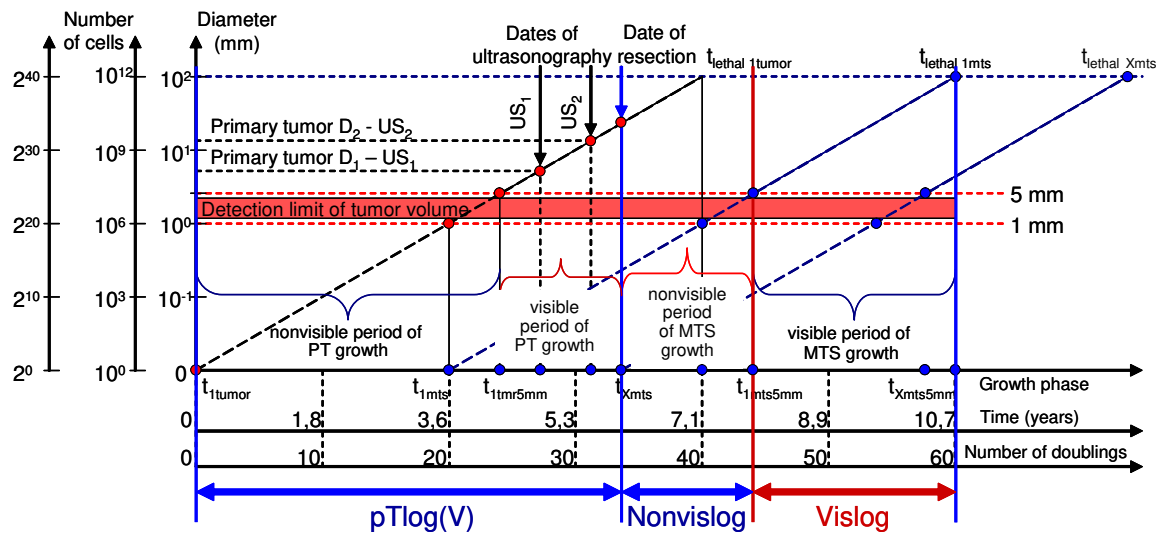

Figure 2: The first MTS cell appears on the $20^{t h}$ doubling of PT

2) the percentage of proliferation cells;

3) the percentage of spontaneous cell loss in vivo and/or migratory tumor cells.

\subsubsection{Limitations}

Model does not describe or explain $[1,17,20]$ :

- reasons for the lack of secondary MTS (M1) in patients with stage IA, IIA, IIB, (T1$4 \mathrm{~N} 0 \mathrm{M} 0$ ) within 25-35 years after resection of PT;

- PT growth with MTS in the regional lymph nodes (T1-4N1-3M0) and primary MTS (T1-4N1-3M1);

\subsection{Consolidated mathematical growth model of PT and primary MTS, IV stage, (CoM-IV)}

Stage IV (T1-4N1-3M1) means that secondary distant MTS exist meanwhile PT is growing (M1 - lungs, bones, liver, etc.) [7,22]. Five-year survival rate of stage IV is about 20-25\% among $\mathrm{BC}$ patients [10]. Unfortunately, the available papers do not offer a mathematical model that describes stage IV of BC, i.e. the simultaneous growth of PT and primary distant MTS.

We propose a new mathematical growth model for PT and primary MTS. The model may help to improve predicting accuracy of BC process using an original mathematical model referred to CoM-IV and corresponding software [26-28].

The CoM-IV rests on CoMPaS and complementing formulas that describes correcting coefficient of primary MTS growth rate $\left(K_{p M t s}\right)$, relating with PT growth rate, tumor volume doubling time of primary MTS $\left(T V D T_{p M t s}\right)$, relating with TVDT of PT [28]:

$$
\left\{\begin{aligned}
p M t s_{\log (m)} & =p T_{\log (V)}-20 \\
K_{p M t s} & =\frac{p M t s_{\log (V)}}{p M t s_{\log (m)}} ; \\
T V D T_{p M t s} & =\frac{T V D T_{p T}}{K_{p M t s}} ;
\end{aligned}\right.
$$




$\begin{array}{lll}p M t s_{\log (m)} & - & \text { the number of primary MTS doublings, corresponding to IV stage; } \\ p T_{\log (V)} & -\quad \text { the number of PT doublings; } \\ K_{p M t s} & \text { correcting coefficient of primary MTS growth rate, relating with } \\ & \text { PT growth rate; } \\ T V D T_{p M t s} & -\quad \text { tumor volume doubling time of primary MTS, relating with Tumor } \\ & \quad \text { volume doubling time of PT }\left(T V D T_{p T}\right) ; \\ p M t s_{\log (V)} & -\quad \text { the number of primary MTS doublings. }\end{array}$

\subsubsection{Limitations}

Model does not describe or explain $[1,17,20]$ :

- reasons for the lack of primary MTS (M1) in patients with stage IA, IIA, IIB, (T1-4N0M0) within 25-35 years after resection of $\mathrm{PT}$

\subsection{Consolidated mathematical growth model of PT and secondary MTS in patients with MTS in lymph nodes, III stage, (CoM-III)}

Stage III (T1-4N1-3M0) means that lymph nodes MTS (N1-3) exist meanwhile PT (T1-4) is growing $[7,22]$. Moreover, patients with lymph nodes MTS have higher growth rate of secondary MTS comparing with patients without lymph nodes MTS. Unfortunately, the available papers do not offer mathematical growth models of stage III of BC that cover growth process of PT and secondary MTS in patients with lymph nodes MTS.

We propose a new mathematical growth model for PT and secondary MTS in patients with lymph nodes MTS. The model may help to improve predicting accuracy of BC process using an original mathematical model referred to CoM-III and corresponding software. Consequently, we are interested in: 1) modelling the whole natural history of PT and secondary MTS in patients with lymph nodes MTS; 2) developing adequate and precise CoM-III that reflects relations between PT and MTS; 3) analysing the CoM-III scope of application; 4) implementing the model as a software tool.

The CoM-III rests upon CoMPaS and complementing formulas [27]:

$$
\left\{\begin{aligned}
s M t s_{\log (V)(N+)} & =p T_{\log (V)}-20 ; \\
K_{s M t s(N+)} & =\frac{20}{s M t s_{\log (V)(N+)} \times 18 \times n}+1 ; \\
T V D T_{s M t s(N+)} & =\frac{T V D T_{p T}}{K_{s M t s(N+)}} ;
\end{aligned}\right.
$$

\footnotetext{
$s M t s_{\log (V)(N+)} \quad$ - the number of doublings of secondary MTS in patients with lymph nodes MTS;

$p T_{\log (V)} \quad$ - the number of doublings of PT;

20 - the number of doublings of PT that coincides with the appearance of the first metastatic cell;

$K_{s M t s(N+)} \quad-\quad$ correcting coefficient of secondary MTS of patients with lymph nodes MTS relating with PT growth rate;

$n \quad-\quad$ the number of affected lymph nodes $(\min =0, \max =$ $18)$;

$T V D T_{s M t s(N+)}$ - tumor volume doubling time of secondary MTS of patients with lymph nodes MTS relating with tumor volume doubling time of $\mathrm{PT}\left(T V D T_{p T}\right)$.
} 


\subsubsection{Limitations}

Model does not describe or explain $[1,17,20]$ :

- reasons for the lack of MTS in lymph nodes (N1-3) in patients with stage IA, IIA, IIB, (T1-4N0M0) within 25-35 years after resection of PT

\section{Results}

The CoMBreC was threefold: CoMPaS (stage I-II), CoM-III (stage III), CoM-IV (stage IV). Tab. 3 demonstrates the results of calculations via CoMBreC.

Table 3: Calculations with data from CoMBreC $+[6]+[6,7]+[30]$

\begin{tabular}{|c|c|c|c|c|c|c|}
\hline & & T1a & T1b & T1c & T2 & T3-4 \\
\hline & \multicolumn{6}{|l|}{ CoMPaS } \\
\hline 1 & $p T_{(D)}$ at surgery $(\mathrm{mm})$ & 4.50 & 8.50 & 15.10 & 28.50 & 64.60 \\
\hline 2 & $p T_{\log (V)}$ & 26.40 & 29.20 & 31.70 & 34.40 & 38.00 \\
\hline 3 & $T V D T_{p T}$ & 70.00 & 70.00 & 70.00 & 65.00 & 60.00 \\
\hline 4 & $K_{s M t s(N-)}(\mathrm{N} 0, \mathrm{n}=0)$ & 1.00 & 1.00 & 1.00 & 1.00 & 1.00 \\
\hline 5 & $T V I$ & 70.00 & 70.00 & 70.00 & 65.00 & 60.00 \\
\hline 6 & $s M t s_{\log (V)}$ & 6.44 & 9.19 & 11.68 & 14.43 & 17.97 \\
\hline \multirow[t]{2}{*}{7} & $s M t s_{D}$ & 0.04 & 0.08 & 0.15 & 0.28 & 0.64 \\
\hline & \multicolumn{6}{|l|}{ CoM-III } \\
\hline 8 & $K_{s M t s(N+)}(\mathrm{N} 1$, & 1.23 & 1.16 & 1.12 & 1.09 & 1.05 \\
\hline 9 & $T V D T_{s M t s}$ & 65.09 & 64.44 & 62.37 & 59.77 & 56.90 \\
\hline 10 & $s M t s_{\log (1}$ & 7.92 & 10.70 & 13.11 & 15.69 & 18.95 \\
\hline 11 & $s M t s_{L}$ & 0.06 & 0.12 & 0.21 & 0.38 & 0.80 \\
\hline 12 & $K_{s M t}$ & 1.69 & 1.49 & 1.37 & 1.26 & 1.16 \\
\hline 13 & $T V D T$ & 47.42 & 50.28 & 51.21 & 51.48 & 51.56 \\
\hline 14 & $s M t s_{(D)}(\mathrm{N} 2$ & 0.12 & 0.24 & 0.40 & 0.67 & 1.25 \\
\hline 15 & $s M t s_{\log (V)}($ & 10.87 & 13.71 & 15.97 & 18.22 & 20.91 \\
\hline 16 & $K_{s M t s}$ & 2.60 & 2.15 & 1.86 & 1.61 & 1.38 \\
\hline 17 & $T V D T_{s M t s(}$ & 30.74 & 34.93 & 37.71 & 40.30 & 43.42 \\
\hline 18 & $s M t s_{(D)}(\mathrm{N} 3$ & 0.48 & 0.96 & 1.50 & 2.17 & 3.10 \\
\hline \multirow[t]{2}{*}{19} & $s M t s_{\log }$ & 16.77 & 19.74 & 21.68 & 23.28 & 24.83 \\
\hline & \multicolumn{6}{|l|}{ CoM-IV } \\
\hline 20 & $K_{p M t s(N+)}(\mathrm{M} 1$ & 4.60 & 3.20 & 2.52 & 2.04 & 1.64 \\
\hline 21 & $T V D T_{p M t s(N+)}(\mathrm{M} 1)$ & 17.40 & 23.40 & 27.80 & 31.80 & 36.70 \\
\hline 22 & $p M t s_{\log (V)}(\mathrm{M} 1)$ & 29.40 & 29.40 & 29.40 & 29.40 & 29.40 \\
\hline 23 & $p M t s_{(D}(\mathrm{M} 1)$ & 9.0 & 9.0 & 9.0 & 9.0 & 9.0 \\
\hline
\end{tabular}

Legend of Tab. 3:

- TNM parameters depend on PT size: T1, T2, T3, T4, N1, N2, N3, M0, M1 [22]:

- row 1 uses data from tables of paper [7];

- row 2 is calculated from row 1 ;

- row 3 uses data from figure of paper [10];

- $p T_{(D)}$ means a mean size (mm) of primary tumor at surgery (resection PT); 
- $p T_{\log (V)}$ means the number of doublings of PT at surgery (resection PT);

- $T V D T_{p T}$ means a mean tumor volume doubling time of PT at surgery (resection PT);

- $\operatorname{sMts}_{(D)}$ means a mean size $(\mathrm{mm})$ of secondary distant MTS at surgery (resection PT);

- $s M t s_{\log (V)}$ means the number of doublings of secondary distant MTS at surgery (resection PT);

- KsMts means a mean correcting coefficient of secondary distant MTS growth rate for N1 (n=1-3), N2 $(\mathrm{n}=4-9), \mathrm{N} 3(\mathrm{n}=10-18)$;

- $T V D T_{s M t s(N+)}$ means a mean tumor volume doubling time of secondary distant MTS for N1 ( $\left.\mathrm{n}=1-3\right)$, $\mathrm{N} 2(\mathrm{n}=4-9), \mathrm{N} 3(\mathrm{n}=10-18)$;

- $\operatorname{pMts}_{(D)}$ means a mean size $(\mathrm{mm})$ of primary MTS at surgery (resection PT);

- $p M t s_{\log (V)}$ means the number of doublings of primary MTS at surgery (resection PT);

- KpMts means a mean correcting coefficient of primary MTS growth rate (M1);

- $T V D T_{p M t s(N+)}$ means a mean tumor volume doubling time of primary MTS growth rate (M1);

For the first time, it was detected that:

- the TVDT of MTS was in range of $\mathbf{6 2 . 9}$ and $\mathbf{6 8 . 2}$ years for pT1, pT2, pT3 considering sizes of PT;

- the TVDT of MTS was in range of $\mathbf{4 5 . 7}$ and $\mathbf{4 8 . 1}$ years for pT4. In other words, the tumor growth aggressively (pT4) considering sizes of PT;

- the whole natural history of PT and MTS growth (the period from the appearance of the first tumor cell up to death) was in range of $\mathbf{1 0 . 3}$ and $\mathbf{1 1 . 2}$ years for pT1, pT2, pT3 (Fig. $3)$;

- the whole natural history of PT and MTS growth (the period from the appearance of the first tumor cell up to death) was in range of $\mathbf{7 . 5}$ and $\mathbf{7 . 9}$ years for pT4.

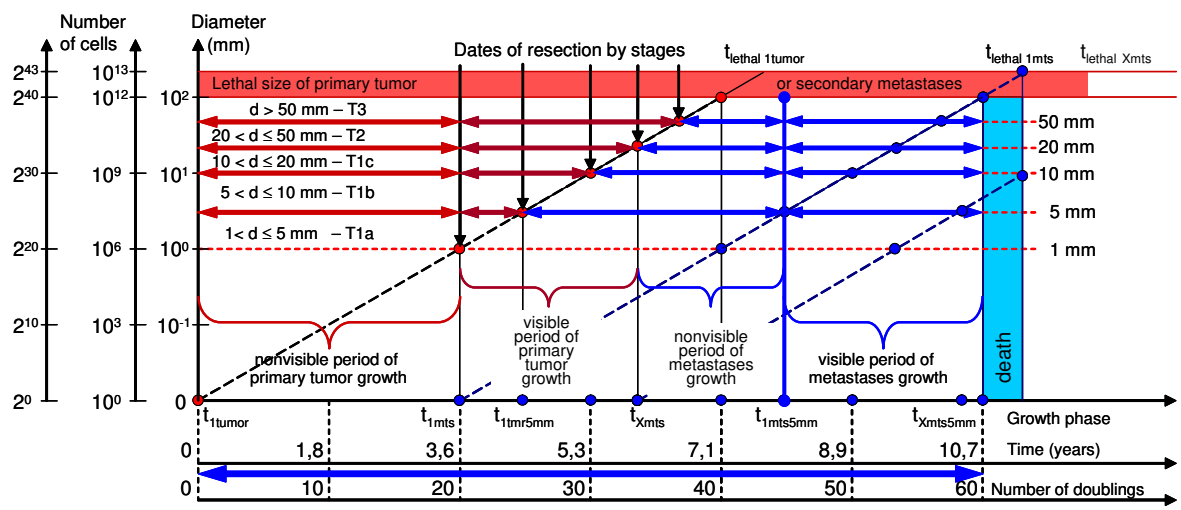

Figure 3: The whole natural history of PT growth - from the appearance of the first tumor cell up to death - in range of 10.3 and 11.2 years for pT1, pT2, pT3

The survival of BC patients included both of the non-visible and visible periods of MTS growth whereas non-visible was the period from date of resection $\mathrm{PT}$ to date of diagnostic of secondary distant MTS 5-10 mm (MTS free period), visible was the period from date of diagnostic of secondary distant MTS 5-10 mm to the death of patient BC.

For the first time, it was detected that the survival was in range of $\mathbf{2 . 9}$ to $\mathbf{3 . 0}$ years for pT1, pT2, pT3 (Fig.3). 
Non-visible and visible periods of MTS growth whereas non-visible was the period from date of resection PT to date of diagnostic of secondary distant MTS 5-10 mm (MTS free period), visible was the period from date of diagnostic of secondary distant MTS 5-10 mm to the death of patient $\mathrm{BC}$.

For the first time, it is possible to calculate an approximate period of appearance of the first MTS cell considering size PT (parameter T) for pT1, pT2, pT3.

For the first time, it was detected that the sizes of secondary distant MTS was in range of $\mathbf{0 . 0 8}$ and $\mathbf{0 . 6 4} \mathrm{mm}$ at date of resection PT. In other words, the size of secondary distant MTS was lower than $1 \mathrm{~mm}$ (the minimum size for diagnostic of tumor), consequently, secondary distant MTS could not be detected (Fig. 4).

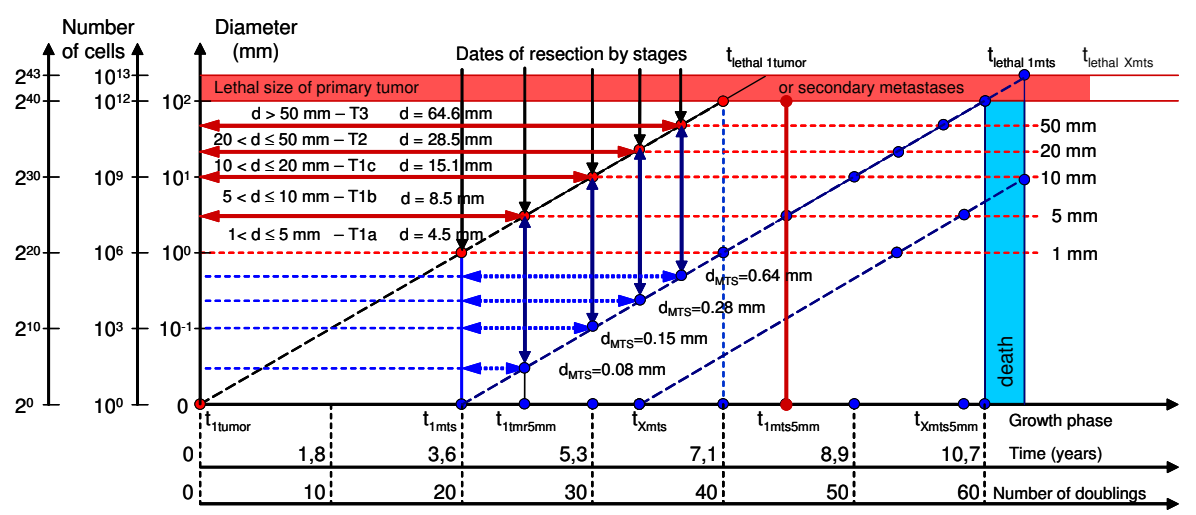

Figure 4: Size of secondary distant metastases was in range of $0.08 \mathrm{~mm}$ and $0.64 \mathrm{~mm}$ at surgery date (diameter below diagnostic level).

For the first time, it was detected that:

- the non-visible period of MTS growth was in range of $\mathbf{1 . 9}$ to $\mathbf{3 . 2}$ years for pT1, pT2, pT3;

- the visible period of MTS growth was in range of $\mathbf{1 . 9}$ to $\mathbf{2 . 1}$ years for pT1, pT2, pT3;

- the visible period of MTS growth was in range of $\mathbf{1 . 8}$ to 1.9 years for pT4. In other words, the visible period of MTS growth for pT4 almost equalled the visible period of MTS growth for $\mathbf{p T 1}, \mathbf{p T 2}, \mathbf{p T 3}$. 
The CoMBreC describes correctly PT and primary distant MTS growth T1-4N0-3M1 stage IV with (N1-3) or without MTS in lymph nodes (N0) (Fig. 5).

The CoMBreC describes correctly T1-4N1-3M1 - stage IV the period of PT growth considering TNM classification (parameter T).

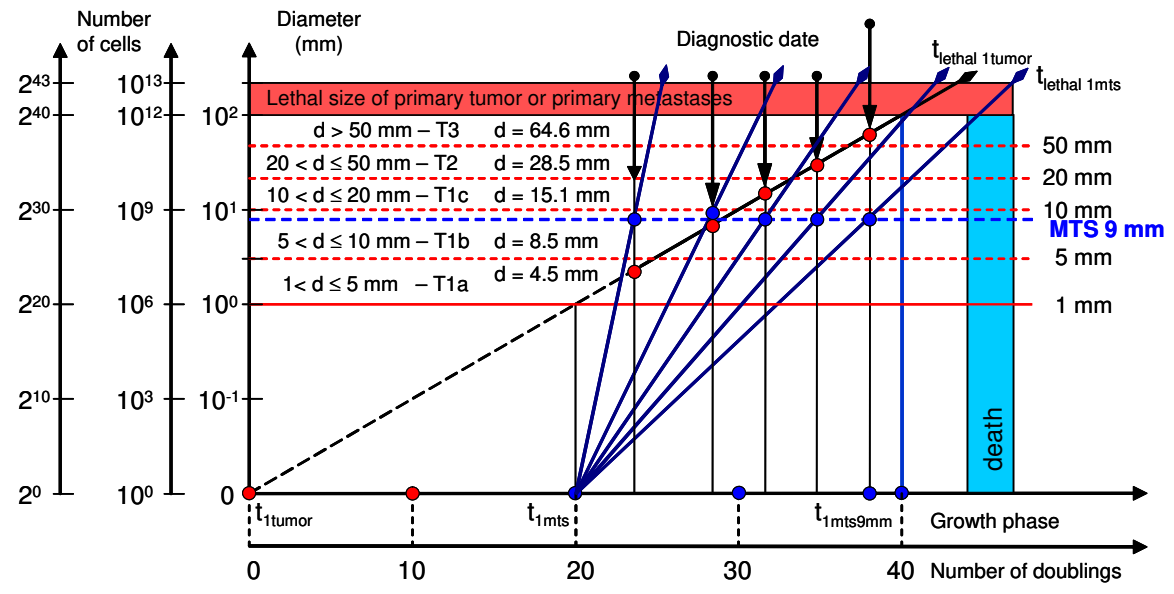

Figure 5: Whole natural history of PT and primary distant MTS of patients with BC (IV stage). Parameter T (T1a: $1 \mathrm{~mm}<\mathrm{d} \leq 5 \mathrm{~mm}$; T1b: $5 \mathrm{~mm}<\mathrm{d} \leq 10 \mathrm{~mm}$; T1c: $10 \mathrm{~mm}<$ $\mathrm{d} \leq 20 \mathrm{~mm}$; T2: $20 \mathrm{~mm}<\mathrm{d} \leq 50 \mathrm{~mm} ; \mathrm{T} 3: \mathrm{d}>50 \mathrm{~mm}$; T4: $\mathrm{d}=$ any). Parameter $\mathrm{N}$ (N0: $\mathrm{n}=0 ; \mathrm{N} 1: \mathrm{n}=1-3 ; \mathrm{N} 2: \mathrm{n}=4-9, \mathrm{~N} 3: \mathrm{n}=10)$ - affected lymph nodes. Parameter M (M1) - distant primary MTS.

For the first time, considering H.R. Withers [33], the CoMBreC allowed us to calculate the variety of correcting coefficients of an increase:

1) the growth rate of secondary distant MTS relating with the growth rate of PT considering stage and sizes of PT (parameter T - pT1a, pT1b, pT1c, pT2, pT3-4) and the number of affected lymph nodes (parametr N, stage III);

2) the growth rate of primary distant MTS relating with the growth rate of PT considering stage and sizes of PT (parameter T - pT1a, pT1b, pT1c, pT2, pT3-4).

The CoMBreC demonstrated changes of the growth rate of secondary distant MTS relating with the growth rate of PT considering stage and sizes of PT (parameter T - pT1a, pT1b, pT1c, pT2, pT3-4) and the number of affected lymph nodes (parametr N, stage III). 
The CoMBreC for T1aN0-3M0-1 (Fig. 6): T1aN0M0 (stage I), T1aN1M0 (stage II) , T1aN2-3M0 (stage III), T1aN0-3M1 (stage IV).

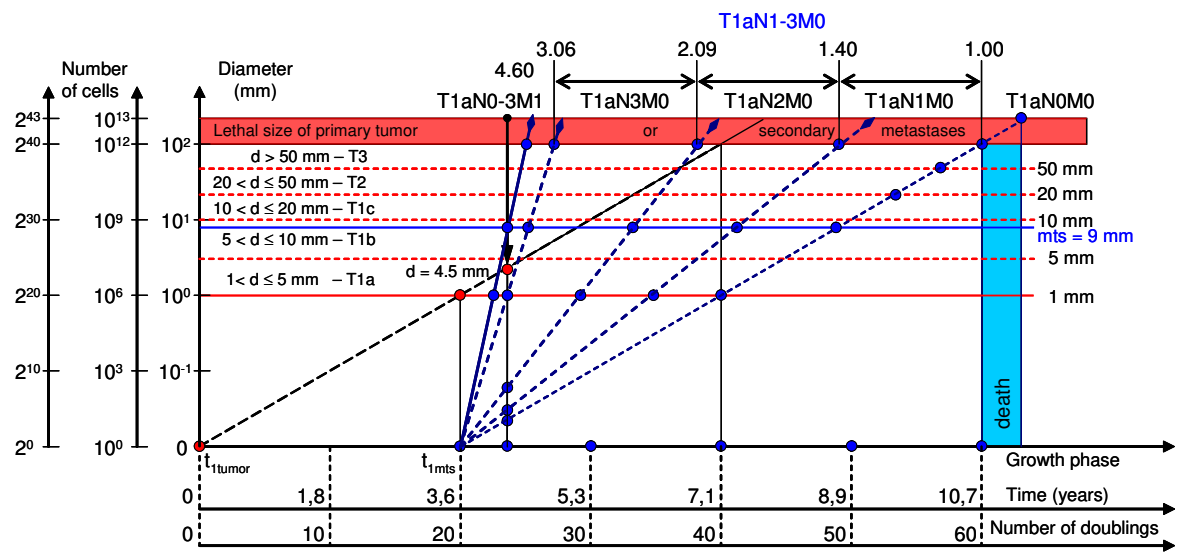

Figure 6: T1aN0-3M0-1. The whole natural history of PT and secondary distant MTS for BC patients. Parameter T (T1a: $1 \mathrm{~mm}<\mathrm{d} \leq 5 \mathrm{~mm})$ - diameter of PT. Parameter N (N0: $\mathrm{n}=0$; N1: $\mathrm{n}=1-3 ; \mathrm{N} 2: \mathrm{n}=4-9, \mathrm{~N} 3: \mathrm{n}=10-18)$ - affected lymph nodes. Parameter M (M0: no evidence of distant MTS; M1: distant detectable MTS).

The CoMBreC for T1bN0-3M0-1 (Fig. 7): T1bN0M0 (stage I) , T1bN1M0 (stage II) , T1bN2-3M0 (stage III), T1bN0-3M1 (stage IV).

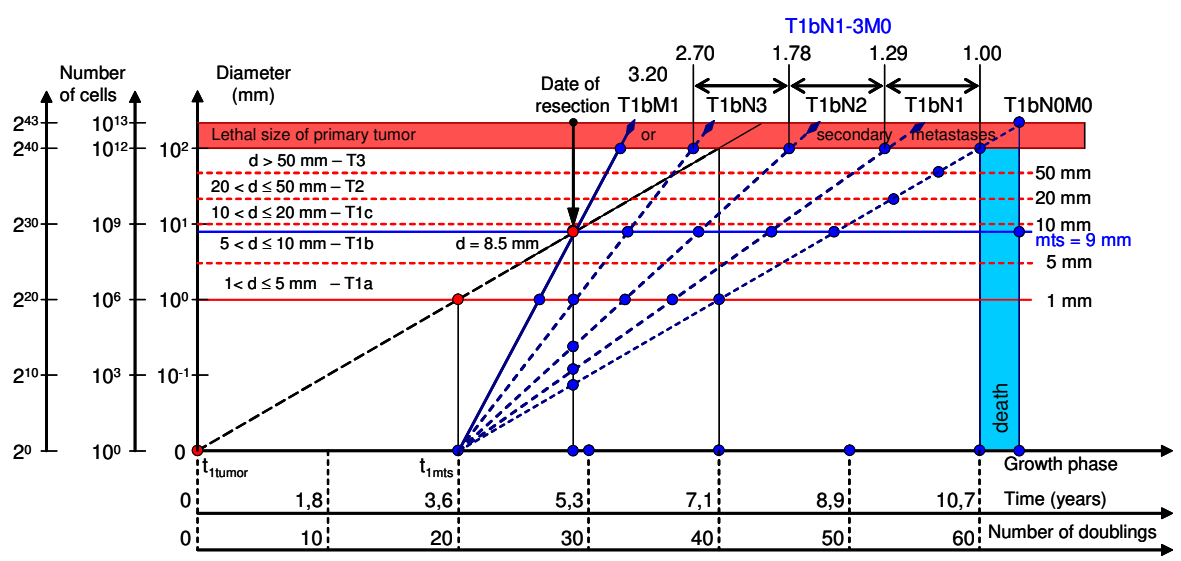

Figure 7: T1bN0-3M0-1. The whole natural history of PT and secondary distant MTS for BC patients. Parameter T (T1b: $5 \mathrm{~mm}<\mathrm{d} \leq 10 \mathrm{~mm}$ ) - diameter of PT. Parameter N (N0: $\mathrm{n}=0$; N1: $n=1-3 ; N 2: n=4-9$, N3: $n=10-18)$ - affected lymph nodes. Parameter M (M0: no evidence of distant MTS; M1: distant detectable MTS). 
The CoMBreC for T1cN0-3M0-1 (Fig. 8): T1cN0M0 (stage I), T1cN1M0 (stage II) , T1cN3M0 (stage III), T1cN0-3M1 (stage IV).

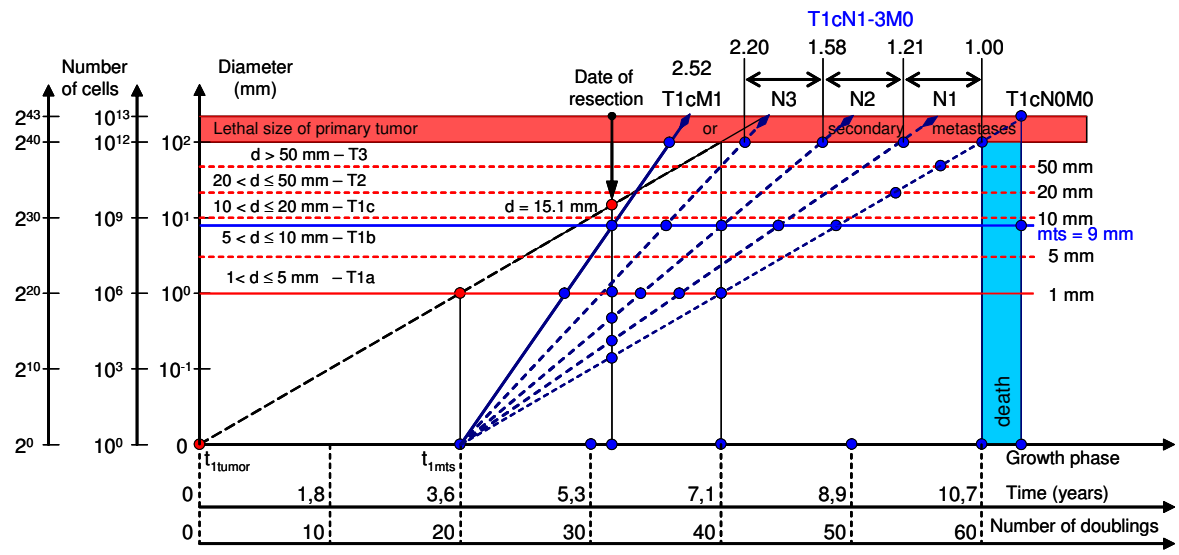

Figure 8: T1cN0-3M0-1. The whole natural history of PT and secondary distant MTS for BC patients. Parameter T (T1c: $10 \mathrm{~mm}<\mathrm{d} \leq 20 \mathrm{~mm}$ ) - diameter of PT. Parameter N (N0: $\mathrm{n}=0$; N1: $\mathrm{n}=1-3 ; \mathrm{N} 2: \mathrm{n}=4-9, \mathrm{~N} 3: \mathrm{n}=10-18)$ - affected lymph nodes. Parameter M (M0: no evidence of distant MTS; M1: distant detectable MTS).

The CoMBreC for T2N0-3M0-1 (Fig. 9): T2N0-1M0 (stage II), T2N2-3M0 (stage III), T2N0-3M1 (stage IV).

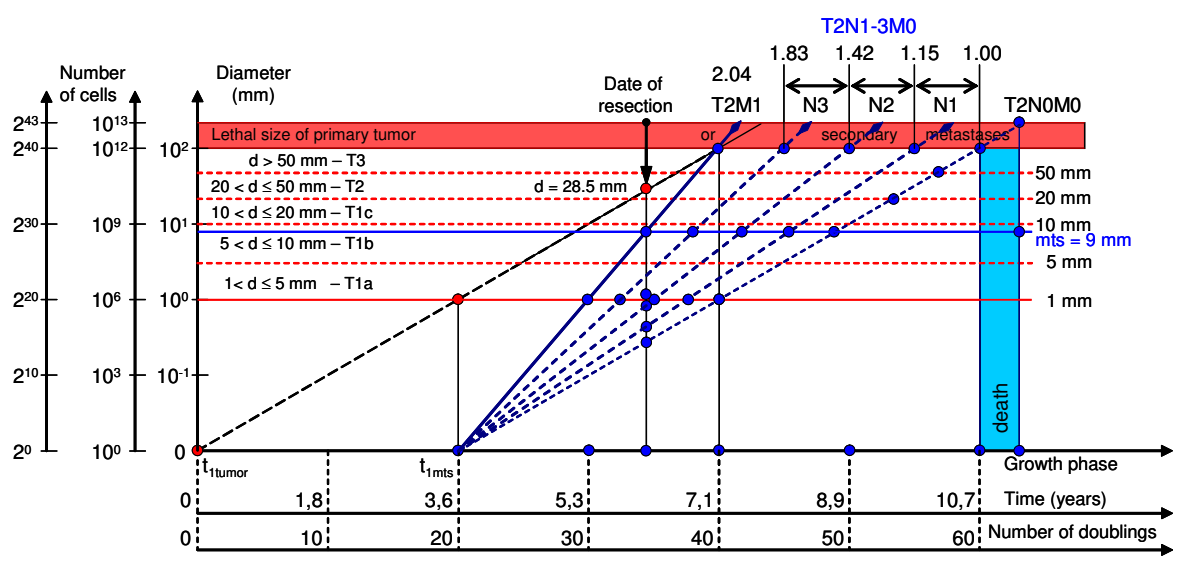

Figure 9: T2N0-3M0-1. The whole natural history of PT and secondary distant MTS for BC patients. Parameter T (T2: $20 \mathrm{~mm}<\mathrm{d} \leq 50 \mathrm{~mm})$ - diameter of PT. Parameter N (N0: $\mathrm{n}=0$; N1: $n=1-3 ; N 2: n=4-9$, N3: $n=10-18)$ - affected lymph nodes. Parameter M (M0: no evidence of distant MTS; M1: distant detectable MTS). 
The CoMBreC for T3N0-3M0-1 (Fig. 10): T3N0M0 (stage II), T3N1-3M0 (stage III), T3N0-3M1 (stage IV).

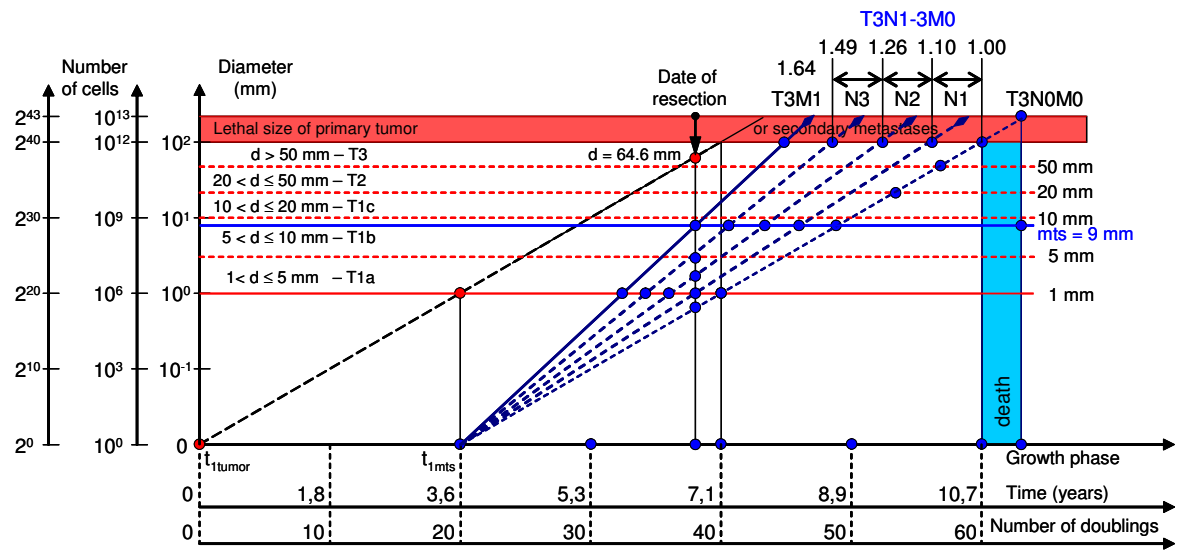

Figure 10: T3N0-3M0-1. The whole natural history of PT and secondary distant MTS for BC patients. Parameter T (T3: $50 \mathrm{~mm}<\mathrm{d})$ - diameter of PT. Parameter N (N0: $\mathrm{n}=0$; N1: $\mathrm{n}=1-3 ; \mathrm{N} 2: \mathrm{n}=4-9, \mathrm{~N} 3: \mathrm{n}=10-18)$ - affected lymph nodes. Parameter M (M0: no evidence of distant MTS; M1: distant detectable MTS).

\section{Software tool for prediction BC developing}

At this stage, it is relevant to shed light on predictor specifications. We implement the CoMPaS, CoM-III and CoM-IV as a software tool. The application is build using Swift and referred as CoMBreC.

$\begin{array}{ll}\text { AppStore Application Description } \\ \text { Developer } & \text { Ella Tyuryumina } \\ \text { Category } & \text { Medicine } \\ \text { Updated } & 5.04 .2017 \\ \text { Version } & 2.0 \\ \text { Size } & 29 \mathrm{MB} \\ \text { Compatibility } & \text { Requires iOS 10+ } \\ \text { Compatible with } & \text { iPhone } \\ & \text { and iPod touch } \\ \text { Languages } & \text { English }\end{array}$
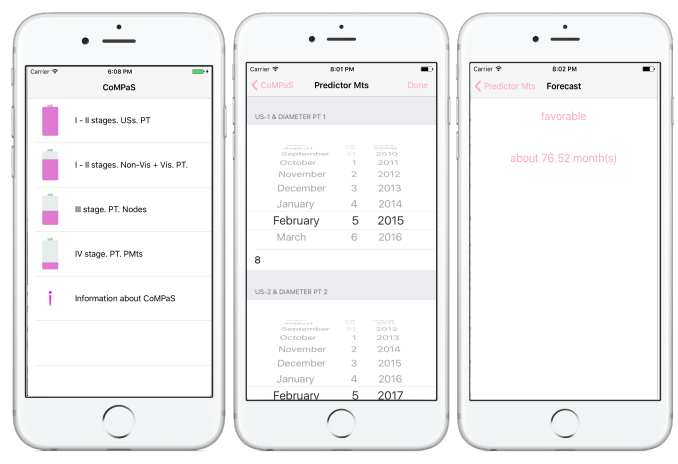
INPUT DATA:

- the first ultrasound diagnostic data:

- date

- diameter $(\mathrm{mm})$

- the second ultrasound diagnostic data:

- date

- diameter $(\mathrm{mm})$

- * diameter of primary MTS (for CoM-IV)

- * the number of affected lymph nodes (n) (for CoM-III)

\section{OUTPUT DATA:}

- forecast:

- the number of months

- category of forecast:

* favorable

* mid-favorable

* unfavorable

\section{The CoMPaS model and predictive software:}

1) allow us to detect different growth periods of PT and secondary distant MTS growth in patients without lymph nodes MTS;

2) allow us to make forecast of the period of secondary distant MTS manifestation in patients without lymph nodes MTS;

3) have higher average prediction accuracy than the other tools;

4) can improve forecasts on survival of $\mathrm{BC}$ and facilitate optimisation of diagnostic tests.

\section{The CoM-III model and predictive software:}

1) allow us to detect different growth periods of PT and secondary distant MTS growth in patients with lymph nodes MTS;

2) allow us to make forecast of the period of secondary distant MTS manifestation in patients with lymph nodes MTS;

3) have higher average prediction accuracy than the other tools;

4) can improve forecasts on survival of BC and facilitate optimisation of diagnostic tests.

\section{The CoM-IV model and predictive software:}

1) allow us to detect different growth periods of PT and primary distant MTS growth;

2) allow us to make forecast of the period of primary distant MTS manifestation;

3) have higher average prediction accuracy than the other tools;

4) can improve forecasts on survival of BC and facilitate optimisation of diagnostic tests.

It is necessary to collect predictions in one database to compare forecasts with real data and estimate effectiveness of proposed model. Consequently, the CoMPaS connects to database that allows to test application and model.

As it turns out, the new predictive tool: 1) is a solid foundation to develop future studies of $\mathrm{BC}$ models; 2) does not require any expensive diagnostic tests; 3 ) is the first predictor that makes forecast using only current patient data, whilst the others are based on the additional statistical data. 


\section{Discussion}

Considering previous studies, the growth rate of primary MTS in BC patients with stage T14N1-3M1 (parameter M1 - stage IV) should be higher than natural growth rate of PT [4-7,9, $10,13,15,21,22]$.

S. Koscielny et al. (1985) [12] have showed the growth rate of secondary distant MTS is higher than the natural growth rate of PT 2.2 times.

M. Tubiana et al. (1989) [25] have proposed that growth rate of secondary distant MTS of $\mathrm{BC}$ could have low and high growth rate. Consequently, growth rate of secondary distant MTS of $\mathrm{BC}$ could be equal or higher than the natural growth rate of $\mathrm{PT}$.

H.R. Withers et al. (2006) [33] have provided an attempt of summarising data of the growth rate of secondary distant MTS and building growth model of secondary distant MTS.

The unique CoMBreC described parameters of $\mathrm{BC}(\mathrm{T}, \mathrm{M}, \mathrm{N})$ according the TNM classification, Fig. 6 - 10. As it should be highlighted, the calculations via CoMBreC corresponded to 10-15 years survival of BC patients. In other words, CoMBreC covers all stages in terms of description growth and prediction development of BC growth process (Tab 4).

Table 4: Clinical Classification of Cancer

\begin{tabular}{|l|c|c|c|c|}
\hline Stage & T & N & M & Model \\
\hline \hline Stage I & T1 & N0 & M0 & CoMPaS \\
\hline Stage IIA & T0 & N1 & M0 & CoM-III \\
& T1 & N1 & M0 & CoM-III \\
& T2 & N0 & M0 & CoMPaS \\
\hline Stage IIB & T2 & N1 & M0 & CoM-III \\
& T3 & N0 & M0 & CoMPaS \\
\hline Stage IIIA & T0 & N2 & M0 & CoM-III \\
& T1 & N2 & M0 & CoM-III \\
& T2 & N2 & M0 & CoM-III \\
& T3 & N1, N2 & M0 & CoM-III \\
\hline Stage IIIB & T4 & N0 & M0 & CoMPaS \\
& T4 & N1 & M0 & CoM-III \\
& T4 & N2 & M0 & CoM-III \\
\hline Stage IIIC & Any T & N3 & M0 & CoM-III \\
\hline Stage IV & Any T & Any N & M1 & CoM-IV \\
\hline
\end{tabular}




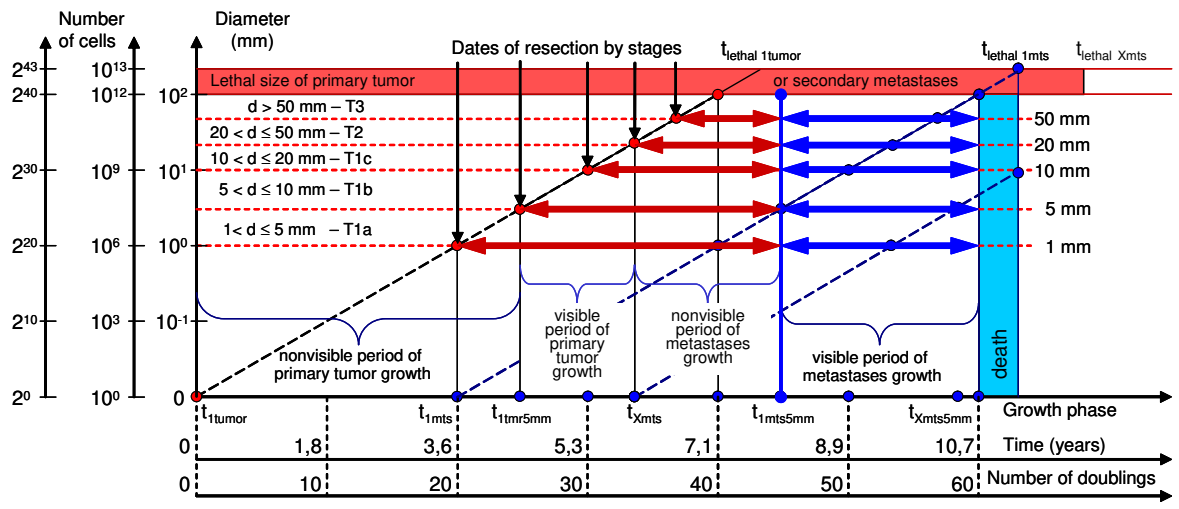

Figure 11: Survival includes the non-visible growth period and the visible growth period of secondary distant MTS.

Relative survival (\%) of patients with BC depends on diameter of PT (T parameter) (Fig. 12), whereas survival (lifetime) means the period between the date of diagnosis (TNM staging system of BC) and the date of a patient death [28-30].

Relative survival (\%) of patients with BC: 1) depends on non-visible growth period of MTS (MTS free period); 2) do not depend on visible growth period of MTS from the first metastasising up to the date of a patient death (Fig. 12).

For the first time, the CoMBreC allowed us to understand the phenomena of differences between 15-years (considering diameter PT) survival and 10-years survival of BC patients (considering date of MTS manifestation) Fig. 12.

The survival included the non-visible period and the visible period of secondary distant MTS. In other words, the survival period was from date of diagnostic secondary distant MTS (5-10 mm) to date of death patient BC (Fig. 6-10,12).

The analysis of non-visible growth period of the secondary distant metastases of breast cancer helps to consider the cause of differences between 10-15-years mortality of breast cancer patients considering the breast cancer stage (parameter M) (Fig. 6-10,12) [26-28]. It is important to mention that the CoMBreC could be used as the standard of estimation the effectiveness of prescribed treatment to a patient BC. In other words, if survival of a patient increases, so the treatment has a positive effect. And vice-versa, if survival of a patient decreases, so the treatment has a negative effect. Consequently, the personalised approach of treatment allows us to increase effectiveness of prescribed treatment to a patient.

The proposed data indicated usage opportunities of the CoMBreC for analysis of real patient data and comparing with statistical data from open sources.

\section{Conclusion}

The analysis of modern state of BC included 34 papers. Meta-analysis of the clinical data showed that growth process of MTS depends on growth process of PT. Given the relation between PT and MTS, the whole natural history of $B C$ was described for each stage to understand the growth process of $\mathrm{BC}$ as a whole, so it allowed us to predict the period of a clinical manifestation of MTS relating to TNM classification. Thus, it was the first time the following were proposed for BC: a) mathematical growth model corresponding to TNM (CoMBreC); b) 
non-statistical software tool for prediction of BC developing.

The "Consolidated mathematical growth Model of Breast Cancer" (CoMBreC) improved the prediction accuracy of BC process by detecting growth periods of PT and MTS for patients with/without lymph nodes. As a consequence, the CoMBreC allowed us to estimate the quality of treatment according to the whole natural history data and patient's survival data. For the first time:

1. The CoMPaS allowed us to calculate:

- the number of doublings for non-visible growth period of secondary distant MTS;

- the number of doublings for visible growth period of secondary distant MTS;

- tumor volume doubling time (days) for non-visible growth period of secondary MTS;

- tumor volume doubling time (days) for visible growth period of secondary MTS.

2. The whole natural history of PT and secondary distant MTS growth was build considering stages (pT1, pT2, pT3 and pT4) and pT sizes.

3. The CoM-III allowed us to:

- calculate correcting coefficients of the growth rate of secondary distant MTS (parameter N1-3, stage III) considering stage (pT1, pT2, pT3 and pT4) and pT sizes;

- show variety of the growth rate of secondary distant MTS (parameter N1-3, stage III) considering stage (pT1, pT2, pT3 and pT4) and pT sizes;

- build whole natural history of PT and secondary distant MTS growth considering PT sizes.

4. The CoM-IV allowed us to:

- calculate correcting coefficients of the growth rate of primary distant MTS (parameter M1, stage IV) considering stage (pT1, pT2, pT3 and pT4) and pT sizes;

- show variety of the growth rate of primary distant MTS (parameter M1, stage IV) considering stage (pT1, pT2, pT3 and pT4) and pT sizes;

- build whole natural history of PT and primary distant MTS growth considering PT sizes.

5. The CoMBreC was implemented as a software tool via mean values from calculations.

6. The CoMBreC was created to help to model different clinical stages of BC (I, II, III and IV) corresponding to TNM classification and to predict 10-years survival of BC patients.

\section{Theoretical Value}

1. Built the consolidated mathematical growth model of primary tumor and secondary distant metastases of breast cancer in patients without lymph nodes metastases, T14 N0M0, stage I-II, (CoMPaS).

2. Determined the critical growth periods of primary tumor and secondary distant metastases of breast cancer to predict survival of patients with breast cancer (CoMPaS). 
3. Built the consolidated mathematical growth model of primary tumor and secondary distant metastases of breast cancer in patients with lymph nodes metastases, T1-4N13M0, stage III, (CoM-III).

4. Built the consolidated mathematical growth model of primary tumor and primary distant metastases of breast cancer, T1-4N0-3M1, stage IV, (CoM-IV).

5. Built the original consolidated mathematical growth model of primary tumor, primary and secondary distant metastases of breast cancer (CoMBreC):

- to describe different clinical stages of breast cancer (I, II, III and IV) corresponding to TNM-classification;

- to predicts the 10-years survival of patients with breast cancer.

\section{Practical Value}

The CoMBreC:

1. The CoMPaS

- was built to predict breast cancer process on stage I-II;

- rested on exponential tumor growth model and consists of a system of determinate nonlinear and linear equations;

- described correctly primary tumor growth (parameter T1-4) and secondary distant metastases growth (parameter M0) in patients without lymph nodes metastases (parameter $\mathrm{N} 0$ );

- determined different growth periods of primary tumor and secondary distant metastases that was not possible beforehand:

- non-visible growth period of primary tumor;

- non-visible growth period of secondary metastases;

- visible growth period of secondary metastases.

- corresponded to data of 10-15-year survival in patients with the different tumor stage;

- facilitated understanding the differences between the nonvisible growth period of MTS and 15-year survival considering tumor stage.

\section{The CoM-III}

- was built to predict breast cancer process on stage III;

- rested on CoMPaS and complementing formulas;

- described correctly primary tumor growth (parameter T1-4) and secondary distant metastases growth (parameter M0) in patients with lymph nodes metastases (parameter N1-3);

- determined different growth periods of primary tumor and secondary distant metastases considering the number of affected lymph nodes:

- non-visible growth period of primary tumor;

- non-visible growth period of secondary metastases; 
- visible growth period of secondary metastases.

- corresponded to data of 10-15-year survival in patients with the different tumor stage;

- facilitated understanding the differences between the nonvisible growth period of MTS and 15-year survival considering tumor stage.

\section{The CoM-IV}

- was built to predict breast cancer process on stage IV;

- rested on CoMPaS and complementing formulas;

- described correctly primary tumor growth (parameter T1-4) and secondary distant metastases growth (parameter M1) in patients with/without lymph nodes metastases (parameter N0-3);

- determined different growth periods of primary tumor and primary distant metastases:

- non-visible growth period of primary tumor;

- non-visible growth period of primary metastases;

- visible growth period of primary metastases.

- corresponded to data of 10-15-year survival in patients with the different tumor stage;

- facilitated understanding the differences between the nonvisible growth period of MTS and 15-year survival considering tumor stage.

4. Software tool via CoMBreC:

- can calculate more accurately MTS free period;

- survival for stages I, II, III, IV of BC considering primary MTS and lymph nodes MTS;

- can estimate a quality of treatment that was prescribed to a patient.

\section{References}

[1] Benzekry, S., Lamont, C., Beheshti, A., Tracz, A., Ebos, J.M., Hlatky, L., Hahnfeldt, P.: Classical mathematical models for description and prediction of experimental tumor growth. PLoS Comput Biol. Vol. 10(8). doi: 10.1371/journal.pcbi.1003800 (2014)

[2] Bloom, H.J., Richarson, W.W., Harries, E.J.: Natural history of untreated breast cancer (18051933). Comparison of untreated and treated cases according to histological grade of malignancy. $\mathrm{Br}$ Med J. Vol. 2(5299). P. 213-221 (1962)

[3] Clare, S.E., Nakhlis, F., Panetta, J.C.: Molecular biology of breast cancer metastasis. The use of mathematical models to determine relapse and to predict response to chemotherapy in breast cancer. Breast Cancer Res. Vol. 2(6). P. 430-435 (2000)

[4] Collins, V.P., Loeffler, R.K., Tivey, H.: Observations on growth rates of human tumors. Am J Roentgen. Vol. 76(5). P. 988-1000 (1956)

[5] Coumans, F.A., Siesling, S., Terstappen, L.W.: Detection of cancer before distant metastasis. BMC Cancer. Vol. 13(283). P. 1-12 (2013)

[6] Engel, J., Eckel, R., Aydemir, U., Aydemir, S., Kerr, J., Schlesinger-Raab, A., Dirschedl, P., Holzel, D Determinants and prognoses of locoregional and distant progression in breast cancer. //Int. J. Radiat. Oncol. Biol. Phys. Vol. 55(5): 1186-1195 (2003) 
[7] Engel, J., Emeny, R.T., Holzel, D. Positive lymph nodes do not metastasize. Cancer Metastasis Rev. Vol. 31(1-2). P. 235-246 (2012)

[8] Friberg, S.: On the growth rates of human malignant tumors: implications for medical decision making. J Oncol. Vol. 55(1). P. 1-22 (2005)

[9] Gullino, P.M.: Natural history of breast cancer. Progression from hyperplasia to neoplasia as predicted by angiogenesis. Cancer. 1977. Vol. 39(6). P. 2697-2703 (1977)

[10] Holzel, D., Eckel, R., Emeny, R.T., Engel, J.: Distant metastases do not metastasize. Cancer Metastasis Rev. Vol. 29(4). P. 737-750 (2010)

[11] Koscielny, S., Tubiana, M., Le, M.G., et al.: Breast cancer: relationship between the size of the primary tumour and the probability of metastatic dissemination. Br J Cancer. Vol. 49(6). P. 709-715 (1984)

[12] Koscielny, S., Tubiana, M., Valleron, A.-J.: A simulation model of the natural history of human breast cancer. Br J Cancer. Vol. 52(4). P. 515-524 (1985)

[13] Koscielny, S., Le, M.G., Tubiana, M.: The natural history of human breast cancer. The relationship between involvement of axillary lymph nodes and the initiation of distant metastases. Br $\mathrm{J}$ Cancer. Vol. 59(5). P. 775-782 (1989)

[14] Laird, A.K.: Dynamics of tumour growth: comparison of growth rates and extrapolation of growth curve to one cell. Br J Cancer. Vol. 19(2). P. 278-291 (1965)

[15] Lee, Y.T., Spratt, J.S.Jr.: Rate of growth of soft tissue metastases of breast cancer. Cancer. Vol. 29(2). P. 344-348 (1972)

[16] Lin, R.S., Plevritis, S.K.: Comparing the benefits of screening for breast cancer and lung cancer using a novel natural history model. Cancer Causes Control. Vol. 23(1). P. 175-185 (2012)

[17] Molina-Pena, R., Alvarez, M.M.: A simple mathematical model based on the cancer stem cell hypothesis suggests kinetic commonalities in solid tumor growth. PLoS One. Vol. 7(2). doi: 10.1371/journal.pone.0026233 (2012)

[18] Norton, L.: A Gompertzian model of human breast cancer growth. Cancer Res. Vol. 48(24). P. 7067-7071 (1988)

[19] Pearlman, A.W.: Breast cancer: Influence of growth rate on prognosis and treatment evaluation. Cancer. Vol. 38(4). P. 1826-1833 (1976)

[20] Rodriguez-Brenes, I.A., Komarova, N.L., Wodarz, D.: Tumor growth dynamics: insights into evolutionary processes. Trends Ecol Evol. Vol. 28(10). doi: 10.1016/j.tree.2013.05.020 (2013)

[21] Schwartz, M.: A biomathematical approach to clinical tumour growth. Cancer. Vol. 14. P. 12721294 (1961)

[22] Shah, R., Rosso, K., Nathanson, S.D.: Pathogenesis, prevention, diagnosis and treatment of breast cancer. World J Clin Oncol. Vol. 5(3). P. 283-298 (2014)

[23] Spratt, J.A., von Fournier, D., Spratt, J.S., Weber, E.E.: Decelerating growth and human breast cancer. Cancer. Vol. 71(6). P. 2013-2019 (1993)

[24] Spratt, J.A., von Fournier, D., Spratt, J.S., Weber, E.E.: Mammographic assessment of human breast cancer growth and duration. Cancer. Vol. 71(6). P. 2020-2026 (1993)

[25] Tubiana, M., Courdi, A.: Cell proliferation kinetics in human solid tumors: relation to probability of metastatic dissemination and long-term survival. Radiother Oncol. Vol. 15(1). P. 1-18 (1989)

[26] Tyuryumina, E., Neznanov, A.: On consolidated predictive model of the natural history of breast cancer considering primary tumour and distant metastases growth. The Breast. ELSEVIER. P. 106$106(2017)$

[27] Tyuryumina, E., Neznanov, A.: On Consolidated Predictive Model of the Natural History of Breast Cancer: Primary Tumor and Secondary Metastases in Patients with Lymph Nodes Metastases. Proceedings of the 2017 International Conference on Digital Health. ACM. P. 60-66. doi $10.1145 / 3079452.3079461(2017)$

[28] Tyuryumina, E., Neznanov, A.: On Consolidated Predictive Model of the Natural History of 
Breast Cancer Considering Primary Tumor and Primary Distant Metastases Growth. Proceedings of IEEE International Conference on Healthcare Informatics. IEEE Computer Society. P. 484-489. doi 10.1109/ICHI.2017.14 (2017)

[29] von Bertalanffy, L.: Quantitative laws in metabolism, growth. Q Rev Biol. Vol. 32(3). P. 217-231 (1957)

[30] von Fournier, D.V., Weber, E., Hoeffken, W., Bauer, M., Kubli, F., Barth, V.: Growth rate of 147 mammary carcinomas. Cancer. Vol. 45(8). P. 2198-2207 (1980)

[31] Vickers, A.J.: Prediction models in cancer care //CA Cancer J Clin. Vol. 61(5). doi:10.3322/caac.20118 (2011)

[32] Weedon-Fekjaer, H., Lindqvist, B.H., Vatten, L.J., Aalen, O.O.and Tretli, S.: Breast cancer tumor growth estimated through mammography screening data. Breast Cancer Res. Vol. 10(3). P. 1-13 (2008)

[33] Withers, H.R., Lee, S.P.: Modeling growth kinetics, statistical distribution of oligometastases. Semin Radiat Oncol. Vol. 16(2). P. 111-119 (2006)

[34] Moiseenko, V.M.: Natural history of breast cancer. Practical oncology. Vol (1). P. 6-14 (2002) 